influencing the management of a large number of patients. It is an opportunity to support whoever is asking the advice. If advice was not sought the patient may have ended up in the acute hospital due to sub-optimal management.

\section{QIP: IMPLEMENTING A PALLIATIVE CARE ELECTRONIC PATIENT PROFILE TO IMPROVE MDT COMMUNICATION AND HANDOVER}

Fiona Richardson. Northumbria Healthcare NHS Foundation Trust

10.1136/spcare-2021-PCC.121

Background and Introduction Northumbria Healthcare Trust uses an electronic patient record called 'Nervecentre' to display admission information. This was often inaccurate and less palliative care focussed. A palliative care profile ('PCU Profile') was created within this software with the aim to improve information sharing and handover.

Methods Usage of 'Nervecentre' and the data within the current 'Medical Profile' were analysed in terms of accuracy and inclusion of palliative care specific outcome measures (phase of illness and Australia-modified Karnofsky Performance Status (AKPS)). Qualitative data was gathered on the current weekend handover process (junior doctors creating a document every Friday). The 'PCU Profile' included relevant information about the patient's admission with designated areas for weekend handover. The MDT completed and edited the profile during the hospital admission. After implementation, the accuracy of the data included was reviewed and feedback gained.

Results 45 'Medical profiles' were analysed which included 19 inpatients over 10 days. None of these profiles had phase of illness or AKPS documented. All included the correct resuscitation status. The diagnosis was accurate in $67 \%$ with an updated management plan in 29\%. 58\% had the correct past medical history. With regards to weekend handover, a move to using 'Nervecentre' was supported with current issues including reliability, clinical governance and efficiency. The 'PCU Profile' led to $91 \%$ of diagnoses being accurate, 100\% of management plans updated, $82 \%$ of profiles stating AKPS and 91\% including phase of illness. Quantitative feedback is ongoing but has been positive especially with regards to weekend handover.

Conclusions Initial data shows improved accuracy of inpatient information leading to more efficient, accurate and therefore safer verbal handovers thus improving patient care. Virtual handover to the on-call team improved ease of information sharing, confidentiality and patient safety. It is hoped more feedback will allow the profile to evolve in future.

\section{THE DEVELOPMENT OF A SUPPORTIVE AND PALLIATIVE CARE LIVER SERVICE WITHIN AN ACUTE HOSPITAL TRUST TO SUPPORT PATIENTS WITH ADVANCED LIVER DISEASE}

Jayne Kennedy, Jenny Wiseman, Susan Binns, Alastair Cairns. Wrightington, Wigan and Leigh Teaching Hospitals NHS Foundation Trust

10.1136/spcare-2021-PCC.122

Patients with advanced liver disease have significant symptom and psychological burdens. Palliative care can improve symptoms and quality of life. Timely advance care planning (ACP) allows patients to prepare for deterioration and facilitate patients dying in the place of their choice.

Method Collaborative working between the Macmillan Supportive and Palliative Care Team (SPCT) and Gastroenterology colleagues developed a Supportive and Palliative Care Liver Service (SPCLS) to meet the needs of patients with advanced liver disease. SPCT engaged with doctors and nursing staff; discussing the service and referral criteria. SPCT joined Gastroenterology ward rounds, alongside joint working between the Alcoholic Liver Disease Clinic and SPCT Clinic. A retrospective audit looked at inpatient referrals to the SPCT for 19 liver disease patients before and 22 patients after the SPCLS.

Results

- Prior to the SPCLS $68 \%$ of patients referred were in the last days of life at time of referral or had a poor prognosis, this reduced to $23 \%$ after the service started. As a consequence most patients $(63 \%)$ were unable to discuss ACP whilst with the SPCLS 73\% could discuss ACP.

- Prior to the SPCLS in only 21\% of cases did the referring ward team recognise and refer their patients for symptom control, compared with $68 \%$ of patients referred for symptom control after the service started.

- Since the SPCLS started the percentage of patients who received psychological support increased from 53\% to $73 \%$.

- Since the SPCLS started the percentage of non-cancer patients referred to the SPCT dying in hospital reduced from $90 \%$ to $50 \%$.

Conclusion SPCLS has facilitated patients being referred to SPCT earlier in their illness; there is an improved awareness of symptoms and the role of SPCT. Earlier referral enabled more patients to discuss ACP and receive psychological support. Since the development of the SPCLS fewer patients died in hospital.

\section{BREAKING BARRIERS AND BUILDING BRIDGES: A SYNERGISTIC FUTURE FOR PALLIATIVE CARE IN LIVERPOOL}

Katherine Rugen, Kate Marley, Julie Bellieu, Kate Dreyer. Woodlands Hospice, University Hospital Aintree

\subsection{6/spcare-2021-PCC.123}

Background and Methods Professionals in Liverpool have designed the IMPaCT (Integrated Mersey Palliative Care Team) model of care to improve access for patients, their families and other professionals. The COVID-19 pandemic enabled implementation of this much more quickly than envisaged. The IMPaCT service in North Liverpool was piloted over July to September 2020 and went 'live' in October. The 'Hub' accepts calls and referrals from anyone and comprises coordinators from hospice outpatient, hospital and community palliative care specialist nursing teams. This single point of access allows for sharing of information, elimination of duplication, and reduces delays in care. Patients are no longer discharged when they move between settings; once they are referred to the IMPaCT service, they remain on the locality caseload until their death.

Results In North Liverpool 136 patients have been regularly reviewed under the newly formed nurse-led surveillance clinic 